\title{
About a New Approach to Control Electron Flow in Unitary Quantum Theory
}

\author{
Leo G. Sapogin \\ Department of Physics, Technical University (MADI) \\ 64 Leningradsky pr., A-319, 125319, Moscow, Russia \\ Tel: 7-499-155-0492Ｅ-mail: sapogin@cnf.madi.ru \\ V. A. Dzhanibekov \\ Department of Cosmophysics, Tomsk State University \\ 36 Lenina st., 634050, Tomsk, Russia
}

Tel: 7-917-530-0487 E-mail: vdzhan42@yandex.ru

V. G. Sapogin

Department of Physics, Taganrog Institute of Technology

Southern Federal University, 44 Nekrasovsky st., GSP-17A, 347 928, Taganrog, Russia

Tel: 7-928-143-7404 E-mail: sapogin@mail.ru

Received: November 2, 2011 Accepted: November 17, 2011 Published: February 1, 2012

doi:10.5539/apr.v4n1p98

URL: http://dx.doi.org/10.5539/apr.v1n1p98

\begin{abstract}
In the Unitary Quantum Theory a probability of tunneling effects is controlled by initial phase. Mathematical model created for this phenomenon uses equations with oscillating charge. This effect is absent in the Ordinary Quantum Mechanics and it may be used for creating of new electronics devices.
\end{abstract}

Keywords: Unitary Quantum Theory, Tunneling effects, Electronic device, Tunneling diode, Control electron flow, a Set of potential barriers, Oscillating charge

\section{Introduction}

In the world literature it is written that tunnel diode was constructed by Japanese researcher L. Esaki. However, at the end of forties a series of articles about "crystadin", constructed by Russian ham Oleg Losev, was published in Soviet popular magazine "Radio". He had used a falling sector of voltage-current characteristic of the contact point between steel wire and homemade crystal of sulfuric iron $(\mathrm{FeS})$. Such diode had been made from the mixture of iron filings and sulfur powder, heated within test-tube. The coked mass obtained then was broken to pieces, from which the suitable crystal had been chosen. That prototype of tunnel diode was used for oscillatory tuned-circuit $Q$-factor excursion in general crystal set. The articles described the way, how it would be done at home. One of the authors did it himself, had being a schoolboy.

In electronics there are two principal ways to control the electron flow:

(1). The control by interception, when a common vacuum electronic triode or a lock (closing device, a field controlled transistor) executes the control of amplified signal with the help of a grid over the number of electrons passed, while the controlling element represents something like a gate within the water flow.

(2). This way of control involves the procedure, in which electrons are slightly accelerated or slowed down with the help of amplified signal, which leads to the velocity being modulated. Then in the course of their movement in free space the faster electrons overtake the slow ones, so there flow's splitting or grouping into space charge's clots occurs. Further, at this density, the modulated bunch of signals interacts with the resonator or with the system with 
slow wave. Such method of control is used in all super-high-frequency devices (SHF-devices): magnetrons, amplitrons, klystrons, TWT, etc.

In this article we propose some different way to control the electron flow, using the tunnel effect. The suggestion is that it is easier to control the phase of electron wave function than to use other control procedures. In fact, it is essentially new and unknown to science, because it has not yet been established, that the tunnel effect depends on the phase of wave function.

Ordinary tunnel diode has semiconductor crystal with two potential barriers.

Interesting phenomena may be observed in the case of potential barriers' series. From the pure qualitative UQT positions (L. Sapogin, Yu. Ryabov, 2011) it is evident that if there are two high, but quite narrow potential barriers, situated at some distance one from another, then the first barrier will be penetrated only by those particles, which have the phase, corresponding to the phase of the particles with very small charge at the moment of the first barrier's reach. In that case the particle will pass through the first barrier. The second barrier will be also passed by the particles, again having the phase, corresponding to the phase of the particles with very small charge in front of the barrier. Such system of two or more periodic barriers results the fact that the flow, monochromatic correlated with phases, will be cut out from the particles' flow with various energies and phases. In cross-section of that flow there will be particles with one phase only. All this will look like the military favorable training: soldiers are marching, keeping the step and its dimension is strictly equal for all soldiers.

There are rather identical considerations relative to barrier's chain within standard quantum mechanics, but from the viewpoint of that theory one can say nothing about the wave's phase and about the physical sense of phenomena observed. Let us consider this quite interesting situation in details. First clear up, how does it happen in accordance with standard Quantum Mechanics?

\section{Problems in the Ordinary Quantum Mechanics}

Consider the problem of particle's passing through the system of two potential barriers described by Dirac's unit-impulse functions and situated at some distance $a$ one from the other. The potential of such system is following (Fig.1):

$$
U(x)=a[\delta(x)+\delta(x-a)]
$$

Assume the particle's flow moving from left to the right. Let us determine the particle's energy $E$ required for passing both barriers. The Schrodinger equation for the wave function is following:

$$
-\frac{\hbar^{2}}{2 m} \Psi^{\prime \prime}+a[\delta(x)+\delta(x-a)] \Psi=E \Psi,
$$

where $m$ is mass of the particle, $\hbar$-reduced Planck's constant.

At once we can write its solution for the area $1(x<0)$ before the barrier, only where the incident wave exists according to the common approach. The solution for the area $2(0<x<a)$ between the barriers contains both waves (right and reversed). The solution for area $3(x>a)$ after the second barrier contains passed wave only. Therefore, we have the following solutions for equation (2):

$$
\begin{gathered}
\Psi_{1}(x)=\exp (i k x), \quad x<0, \quad k=\sqrt{\frac{2 m E}{\hbar^{2}}}>0, \\
\Psi_{2}(x)=A \sin (k x)+B \cos (k x) \quad 0<x<a, \\
\Psi_{3}(x)=C \exp [i k(x-a)] \quad x>a,
\end{gathered}
$$

The continuity of the wave function and discontinuous character of derivative in points $x=0, x=a$ leads to equalities:

$$
\begin{gathered}
\Psi^{\prime}(+0)-\Psi^{\prime}(-0)=\frac{2 m a}{\hbar^{2}} \Psi(0) \\
\Psi(+0)=\Psi(-0)
\end{gathered}
$$


Joining the wave functions and their derivatives in the points $x=0$ and $x=a$ in standard way and taking into account the above equalities, we get the system of four algebraic equations:

$$
\begin{gathered}
B=1 \\
k A-i k=\frac{2 m a}{\hbar^{2}} \\
A \sin (k a)+B \cos (k a)=C \\
i k C-k A \cos (k a)+k B \sin (k a)=\frac{2 m a C}{\hbar^{2}}
\end{gathered}
$$

The given system is predefined and has solution only under following condition:

$$
\operatorname{tg}(k a)+\frac{\hbar^{2} k}{m a}=0
$$

If $k_{1}, k_{2, \ldots}$ are the roots of this equation, then using the expression for $k$ (has been written at the beginning of this sect.), we are able to determine the energy values, at which the particles penetrate (we say "tunnel") two-barrier's system:

$$
E_{s}=\frac{\hbar^{2} k_{s}^{2}}{2 m}, s=1,2, \ldots,
$$

From the solution of transcendental equation (3) it is obvious that periodic dependence in energy, while tunneling two barriers, appears due to tangent curve to be periodically crossed by straight line, outgoing at some angle from the origin of coordinate system. It is obvious that barriers will be passed by the particles with de Broglie wavelength, being multiplied to $a$. This phenomenon bears a strong resemblance to processes, appearing in the cases of anti-reflecting optic lenses.

\section{Problems in the Unitary Quantum Theory and New Electronic Devices}

We should note an interesting circumstance. If the same problem would be solved in other order that had been first to determine the portion of the particles flux penetrated (tunneled) the barrier and to consider passed portion as incident flux in respect to the second barrier, the result would be absolutely different. The multiplication of two exponents to be given by each barrier just (immediately) suppresses everything. It is very difficult to understand such double game directive for an unprejudiced physicist with mentality non-perverted by "quantum" logic.

There is one more amazing consideration. The particle has not penetrated the barrier but just going to tunnel it or being repulsed, however its "decision" depends on the second barrier distance. But how could it know what is waiting further and what is the second barrier distance? Does the second barrier exist at all? Here we can recollect the perfect words of R. Feynman: May be the particle "sniffs out" the second barrier? And again it is violence over the logic and the mind.

Similar phenomena, but in more tangible and totally understandable form, will take place, if we analyze the solutions of equation with oscillating charge (L. Sapogin, Yu. Ryabov, V. Boichenko, 2005, 2008; L. Sapogin, Yu. Ryabov, 2011).

The equations named "Equations with oscillating charge" are the important elements of our Unitary Quantum Theory (UQT). There are two forms (non-autonomous and autonomous) of these equations. For the first time, non-autonomous equation was simply postulated in references of (Sapogin, 1994a, 1994b, 1996), where this equation was used for description of cold nuclear fusion process due to mutual deuteron interaction. This equation has the following form:

$$
m \frac{d^{2} \mathbf{r}}{d t^{2}}=-2 Q \operatorname{grad} U(\mathbf{r}) \cos ^{2}\left(\frac{m t}{2 \hbar}\left(\frac{d \mathbf{r}}{d t}\right)^{2}-\frac{m \mathbf{r}}{\hbar} \frac{d \mathbf{r}}{d t}+\varphi_{0}\right),
$$

where $m$ is the mass, $\mathbf{r}$ is the radius vector, $U(\mathbf{r})$ is the external potential, $\varphi_{0}$ is the important parameter, called "initial phase", and $Q$ is the average charge of particle. 
The heuristic premises to this equation were following. At first, integral-differential equation of UQT was obtained by L.G. Sapogin (Sapogin, 1979, 1980). Then simplified scalar integral-differential version of equation was solved in (L. Sapogin, V. Boichenko, 1988, 1991). This solution describes periodically appearing and vanishing wave packet, identified with a particle. The bilinear integral of such wave-packet over the whole volume turned out to be equal to the value of dimensionless elementary electric charge with the precision up to $0.3 \%$. It was easy to associate such wave-packet with the simple oscillation of space electric charge that has double charge amplitude, i.e. with oscillating point charge, described by a general Newton equation, but taking into consideration the changes of point's characteristics within the process of movement. In the essence, it is simply the next step in the ordinary motion equation for material point. It is not a new idea for ordinary mechanics. There are well known equations of I. Meshchersky (Mestschersky) for the motion of variable mass bodies and equations of K. E. Tsiolkovsky for rockets' motion.

To our regret, the numerical modeling is embarrassed in the case of barriers system (1), described by delta functions. That is why we have replaced equation (1) by the sum of two Gauss "bells":

$$
U(x)=U_{0}\left[\exp \left(-\frac{x^{2}}{\sigma^{2}}\right)+\exp \left(-\frac{(x-a)^{2}}{\sigma^{2}}\right)\right]
$$

One-dimensional equation with oscillating charge, describing the particle's motion and corresponding to last potential, has been solved numerically in autonomous and non-autonomous cases. As far as the results obtained are slightly different, we have shown them separately, with further comparison.

\subsection{An Autonomous model}

One-dimensional motion equation has the following form:

$$
m \frac{d^{2} x}{d t^{2}}-4 U_{0} Q\left[\operatorname{xexp}\left(-\frac{x^{2}}{\sigma^{2}}\right)+\operatorname{xexp}\left(\frac{(x-a)^{2}}{\sigma^{2}}\right)-\operatorname{aexp}\left(\frac{(x-a)^{2}}{\sigma^{2}}\right)\right] \frac{\cos ^{2}(\varphi)}{\sigma^{2}}=0,
$$

where

$$
\varphi=-\frac{m}{\hbar} \frac{d x}{d t} x+\varphi_{0}
$$

$x=x(t), \varphi_{0}$ - the initial phase, $a$-the distance between the barriers; $\sigma, U_{0}$ are the width and the height of the barrier respectively; $Q, m$ are the average part of particle's charge and mass respectively. The equation has been solved numerically by Runge-Kutta-Merson method. There the numbers of particles tunneled with respect to initial velocity and to initial phases, uniformly distributed in interval from 0 to $\pi$ have been calculated.

The following starting data were used: $Q=1, m=1, \hbar=1, U_{0}=5, a=4, \sigma=1 / 8, V_{0}=1-3.5$. We computed variants for 101 values of initial phase (the case of $\varphi_{0}=\frac{\pi}{2}$ was excluded from calculations) for each initial velocity value. The total number of particles equals to 20502. The results of calculations are shown in the Fig. 2, Fig. 3. The relation between numbers (percentage) of particles and initial velocity (Fig. 2) can be well approximated by simple exponent. The distribution of particle's number in respect of the velocity after passing two barriers (Fig. 3) shows the resonance effect.

From the other side, there exists particles' grouping in respect of velocity as it has been described in the beginning of that section and expected from the most general considerations. It can be seen in Fig. 3, where $x$-axis indicates particles' velocities and $y$-axis indicates the number of particles. It is curious that within velocities' intervals $[1 \div 2.27]$ and $[3 \div 3.25]$ there are no particles at all - forbidden zones. We will discuss it in details later.

\subsection{Non-autonomous model}

Non-autonomous equation has the form of equation (4), but with the other expression of $\varphi$ :

$$
\varphi=\frac{m t}{2 \hbar}\left(\frac{d x}{d t}\right)^{2}-\frac{m}{\hbar} \frac{d x}{d t} x+\varphi_{0}
$$


The numerical integration has been made by Runge-Kutta-Merson method, using following data: $Q=1, m=1$, $\hbar=1, U_{0}=5, a=4, \sigma=1 / 8, V_{0}=1.5-4$. The full number of particles is $\mathrm{N}=20502$. Now, as it can be seen from Fig.4, the expected resonant dependence of barrier's tunneling upon the initial velocity exists. But this resonance effect is slightly suppressed because tunneling probability increases with the velocity thus compensates the drop in probability at moving away from resonance point (horizontal steps at the curve). We have plotted the distribution of particles' exit velocity after tunneling (Fig. 5). It is evident from the plot that forbidden zones are outlined within the area of velocity, which equals 2 . Also, the particles' grouping in respect of velocities may be seen.

With the help of numerical integration of the same equation we have also calculated the probability of barrier tunneling with respect to distance between the barriers $a$, to fixed initial velocity of particles, and to initial phases, uniformly distributed from 0 to $\pi$. Starting data have been following:

$$
Q=1, m=1, \hbar=1, U_{0}=5, a=0.25-12.5, \sigma=1 / 8, V_{0}=2.5 .
$$

Total number of particles is $N=20502$. The dependence obtained may be estimated as expected from quite general viewpoint. Really, the first barrier is to be passed by all particles which phase is so that the particle charge in front of the barrier is small. If further there is second barrier on particles' way, then it would be penetrated without any problem by particles, which have the phase, again corresponding to the phase of the particles with small charge in front of the barrier. If the distance between the barriers has been changed, then the tunneling should be periodically changed. It is illustrated with Fig. 6.

In general, the character of solutions for both types of equations is similar. There the forbidden zones are appeared. But the most interesting fact is that in comparison with initial velocity both, some increasing and some decreasing, velocities are observed. Thus, it can be seen on Fig. 3 that there are many particles with the velocity a little bit more than initial maximum velocity, which is equal to 3.5. We can see the same phenomenon on Fig. 5, where one can find quite enough particles with the velocity more than 4 - initial maximum velocity. It is also evident that in both cases there are many particles with velocity less than the minimal initial one. In any case we will not think that some particles give up their energy to other particles and reduce their velocity and vice versa, what is a cause of Maxwell-Boltzmann statistical distribution. The modeling is to be made each time for one particle only (!) and equation does not mean any interaction with the other particles. We would like to think that additional energy of ensemble obtained, owing to fast particles, is just exactly equal to the energy has been lost by slow ones. Of course, it is pure aesthetic consideration which etymology descends from atavistic nostalgia in conservation law, but we have not checked this circumstance. Besides, such reasons are based on energy and momentum conservation laws that are not fulfilled for both equations (L. Sapogin, Yu. Ryabov, 2011). It is evident that in that case there are no conservation laws for single events at all. These laws appear only after averaging over a large ensemble of events. As the matter of fact it can be easily shown that classical mechanics is obtained from quantum one after summation over a large number of particles.

The unitary quantum theory predicts a number of new phenomena that occur when charged particles pass through a potential barrier. A new type of semiconductor devices, have been based on (L. Sapogin, Yu. Ryabov, V. Boichenko, 2005, 2008; L. Sapogin, Yu. Ryabov, 2011), can be created.

The above equation describes relationship between the particles, passing through the potential barrier, and phases of the wave function. In other words, if the charge of particle that approaches to the barrier is small, this particle passes through the barrier quite easily. Due to this small energy, deuterons can approach each other and interact, but this effect takes place only within a narrow phase's range. This is the explanation of the Cold Nuclear Fusion (CNF). But CNF is extremely improbable process in the Ordinary Quantum Mechanic (L. Sapogin, Yu. Ryabov, 2011)!

In order to describe the particle's behavior in passing through a periodic chain of potential barriers we shall use the most elementary potential of the kind:

$$
U(x)=E x+A \sin ^{2}(x)
$$

Then the motion equation for particle within such potential with superposition of weak uniform field (external operational field) will assume the following form: 


$$
\frac{d^{2} x(t)}{d t^{2}}=(E+A \sin (2 x(t))) \cos ^{2}\left(\frac{t}{2}\left(\frac{d x(t)}{d t}\right)^{2}-x(t) \frac{d x(t)}{d t}+\phi_{0}\right),
$$

where $E$ is the small external operational voltage (power supply).

Assume that a certain number of charged particles with uniform phase and Maxwell velocity distribution move through a periodic chain of potential barriers, being exposed to external electric field. According to the UQT, almost all particles, which have passed through the barriers, have approximately equal velocities and phases (a coherent flow). It is interesting that slow particles are accelerated while the fast ones are slightly slowed down. Consequently, the particle's phase changes too. To check this point the following problem has been set: upon a sequence of five barriers (Fig.7) a flow of particles is directed, the particles possess various velocities (uniform distribution with respect to velocities) and various initial phases, uniformly distributed in $0 \ldots \pi$ region.

In practice this problem has been solved with a mathematical program in two cycles. The first velocity-cycle has contained a phase cycle (101 phase-values have been used) in $0 \ldots \pi$ range. The number of phase values should always be odd, as even in splitting, the point $\frac{\pi}{2}$ is sure to emerge and the PC would hover for many hours until it has reach zero because of equation's singularity in this point. The velocity range has been split into 500 intervals (stretches). Thus, the particle's motion equation has been solved by the Runge-Kutt method of the fourth order $500 * 101=50500$ times, the procedure takes no less than a month, when using ordinary PC.

In fact, the standard Monte-Carlo procedure was applied within two cycles. If the particle changed its velocity sign (was reflected), its behavior was considered no longer and it was excluded from the analysis. All trajectories have been calculated, the histograms of the particles' number distribution in relation to their velocity have been made. These results are given on the plot (see Fig.8).

As it is seen from the plot (Fig.8) a lot of particles, having passed through 5 barriers, possess identical velocities and phases. It is clear that such automatic phase and velocity phasing in periodic potential sharply raises the probability for the deuterons to approach each other, which itself serves as an additional argument to Cold Nuclear Fusion explanation (Fleischmann, 1989; L. Sapogin, 1983, 1994a, 1994b, 1996). Of course, in real lattice this effect is much weaker, because the problem being solved is one-dimensional. In order to solve three-dimensional lattice model (pattern) one needs a very long time and a very powerful supercomputer.

The experiments on the tunnel-effect dependence upon the wave function phase should be carried out by all means. Such a problem had never been considered in Quantum Mechanics because the wave function square module, rather than the wave function itself, has the physical meaning and, hence, the wave function phase has been excluded from the analysis. If the relationship between the particle passage through a potential barrier and the wave function phase will be proved experimentally it will serve as a crucial evidence of the UQT validity and will allow the creating of electronic devices based on the new electron-control principle.

\subsection{A new electronic devices}

Let us regard the operational principle of such new device. Its schematic diagram is given on Fig.9.

This crystal of semiconductor includes several equidistant potential barriers, produced by introducing of impurities. If we vary the potential at the 3-rd barrier, then we vary the phase of approaching to the 3-rd barrier electrons and, consequently, we vary the probability of tunneling. The processes that are to take place within such device are easily predicted: all electrons with equal phases but different energies will pass through the 1 -st barrier. The electrons of de Broglie wave length, equal to the distance between two barriers divided by $N$ (where $N$ is an integer number), will also pass the 2-nd barrier. The energy deviation being rather small, a mono-energetic equal-phase (coherent) electron flow will be formed upon passing through the second barrier. Consequently, any change as it has been potential between the 2-nd and the 3-rd barriers, will cause the change of electron phase at approaching the 3-rd barrier and, hence, the amount of electrons, having passed through the third barrier, will decrease.

The above-predicted results had been simulated on the same 5-barrier chain, as it was the grid being placed between the 4-th and the 5-th barriers, which did not bring about any essential changes of situation. The resulting current behind each barrier had been summed up. In the current value calculation each particle's velocity and instant charge have been taken into consideration. 
In fact, mathematical simulation was carried out for 1-, 2-, 3-, and 5-barrier tunnel diode. Each behind-barrier current's dependence upon the squared velocity of incident particles (this value being proportional to the device operational voltage) is shown at the plots in (Fig.10).

It is clearly seen, that within $[5 \div 10]$ resulting range of volt-ampere properties there is a place with a negative resistance, and in general, these characteristics give a sufficiently accurate description of the tunnel diodes (Fig.10).

Further on, mathematical simulation of a new kind of the device has been made, the concept of the latter being presented on Fig.10 and its operational principle being described above. To achieve this, the velocity of each electron, that having passed through the 4-th barrier, was changed by $5 \%$ as had been compared with its calculated value, the charge's instant character being taken into account. In other words, an attempt was made to imitate the process so as it was the grid, which either accelerated or slowed down by $5 \%$ the electrons that had passed the 4-th barrier. The current that had got through the 5-th barrier was also calculated. The results are given on a summary plot (Fig.10), where two curves, representing positive and negative one correspondingly, as it has been the grid voltage, are shown. Of course, practically control signal must be connected with 3 barriers. The simulation's outcome has surpassed our most far-reaching expectations although it looks much more complicated than has been expected, proceeding from the above theoretical reasoning. The simulation analysis shows that there are some voltage areas, where the gain coefficient is very high; therefore, doubtless, devising of such instruments holds very much promise.

Some strange things are also observed: the current through the 2-nd, 3-rd and 5-th barriers may be bigger than that through the 1-st barrier at the certain voltage values. Any researcher can ask a legitimate question: how can it be possible within a series resistor circuit with one and the same current flowing? The answer to this strange paradox is rather simple: the phases of all electrons, being passed through the chosen point, are such that their summed up charge varies only slightly, which leads to various current values at different points. Note, that just the same phenomenon is arranged by Nature within the so-called "Lecher wires".

It is experimentally established that within the Lecher wires (long lines) specific points exist. There are some points, where the voltage is equal to zero, and other points, where the current is equal to zero. So, the lines can be short circuited in one points without any change of voltage on a payload at the line's end. Similarly, the wire can be safely cut without any damage done in the other points, where the currents that run through the wire, are equal to zero.

Besides, the plot (Fig.10) reveals another curious phenomenon: it is seen that all curves have common intersecting points at voltage values of 1.6 and 6.4. This is a consequence of a trivial resonance effect, when the de Broglie wave length changes by 2 times. In so doing, as the phase had altered by $2 \pi$, nothing will change in the probability for the electron to pass through the barriers via these points.

For example, as opposed to klystron, there is no need to wait a long time till modulated signal will bunches electrons by the velocity into the clots in free space, so the transit time is reduced. Therefore, such a device could apparently permit to obtain very prompt work. Probably, it would be expedient to develop this new semiconductor device on the basis of either gallium arsenide $(G a-A s)$ or aluminum and gallium arsenide ( $A l-x$ - $G a-x-A s)$ super lattice devices, proposed by (Esaki L., Tsu R., 1969). It could also be achieved on the basis of common tunnel diodes or resonance-tunnel dipole transistor (M. Schur, 1990). This experiment could be carried out in institutions possessing sub-micron semiconductor technology. It is also necessary to make a super pure semiconductor device with the electron free path length, being greater than device's dimensions. So, the electron flow's control devices seem to be quite possible and new electronic devices, using the phase control mechanism, can be made. The consequences of these developments are to be very far-reaching.

\section{Acknowledgements}

The authors are thankful for support and effectual discussions of the work to Professors V.M. Dubovik (Dubna, JINR), A.S. Bogomolov, P.I. Pospelov, V.M., Prihod'ko, Yu.A. Ryabov. The authors are also thankful to translator L. K. Sapogina for translation of our work.

\section{References}

Esaki L., \& Tsu R. (1969). Superlattice and Negative Conductivity in Semiconductors. IBM J. Res. Note, RC-2418, March.

Fleischmann M., \& Pons S. (1989). Electroanal. Chem., v.261, p.301. http://dx.doi.org/10.1016/0022-0728(89)80006-3 
Sapogin L. G. (1979). On Unitary Quantum Mechanics. Nuovo Cimento, vol.53A, No 2, p.251. http://dx.doi.org/10.1007/BF02776417

Sapogin, L. G. (1980). A Unitary Quantum Field Theory. Annales de la Fondation Louis de Broglie, vol.5, No 4, p. $285-300$.

Sapogin L. G. (1983). Techniques to youth, Journal, No.1, p.41. (in Russian).

Sapogin, L.G., \& Boichenko V. A. (1988). On the Solution of One Non-linear Equation. Nuovo Cimento, vol.102B, No 4, p.433. http://dx.doi.org/10.1007/BF02728514

Sapogin, L.G., \& Boichenko V. A. (1991). On the Charge and Mass of Particles in Unitary Quantum Theory. Nuovo Cimento, vol.104A, No 10, p.1483.

Sapogin L. G. (1994a). Deuteron Interaction in Unitary Quantum Theory, \& On the Mechanisms of Cold Nuclear Fusion. In: Proceedings of the Forth International Conference on Cold Fusion, vol.4. Theory and Special Topics Papers TR-104188-V4, July 1994, p.171-178, Hawaii.

Sapogin L. G. (1994b). Deuterium Interaction in Unitary Quantum Theory, and On the Mechanisms of Cold Nuclear Fusion. In: Fusion Source Book. International Symposium on Cold Nuclear Fusion and Advanced Energy Sources, Belarussian State University, Minsk, May 24-26, p.91-98.

Sapogin L. G. (1996). Cold Nuclear Fusion and Energy Generation Processes in Terms of the Schroedinger Equation. Chinese Journal of Nuclear Physics, vol.19,\#2, p.115.

Sapogin L. G., Ryabov Yu.A, Boichenko V. A. (2005). Unitary Quantum Theory and a New Sources of Energy, Archer Enterprises, Geneva, NY, USA.

Sapogin L. G., Ryabov Yu. A., \& Boichenko V. A. (2008). Unitary Quantum Theory and a New Sources of Energy, Ed. Science-Press, Moscow, (Russian, transl. from English).

Sapogin L. G. (2010). About Unitary Quantum Field Theory. Applied Physics Research, vol. 2, No 2, p.114-140, November.

Sapogin L. G. (2011). An Unitary Unified Quantum Field Theory. Global Journal of Science Frontier Research, Vol. 11, Issue 4, Version 1.0,p.46-74, July.

Sapogin L.G., \& Ryabov Yu. A. (2011). Approximation Equations with oscillating charge in Unitary Quantum Theory and its applications to the analysis of some quantum problems. International Journal of Applied Science and Technology, Vol.1, No. 5, September.

Sapogin L. G., Dzhanibekov V. A., \& Sapogin V. G. (2011). A new approach to control electron current in Unitary Quantum Theory. International Journal of Applied Science and Technolog,Vol.1, No.6, December 15.

Michael Shur. (1990). Physics of Semiconductor Devices, Prentice-Hall International, Inc.

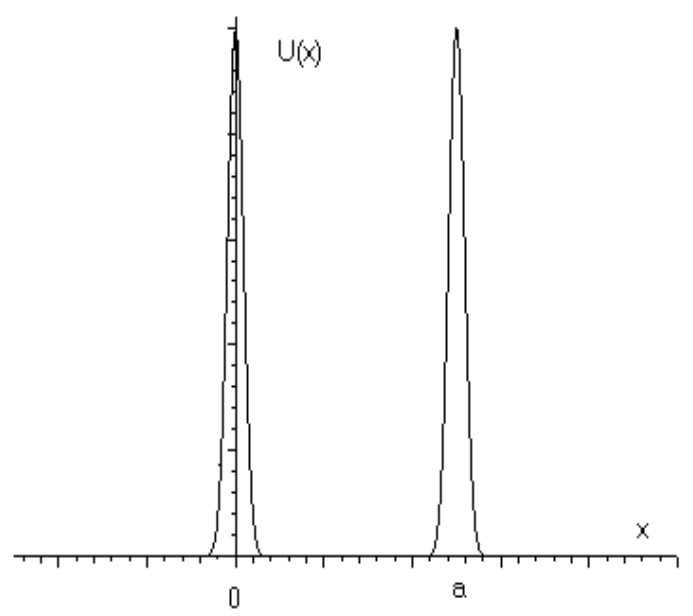

Figure1. Potential of system with two-barriers 


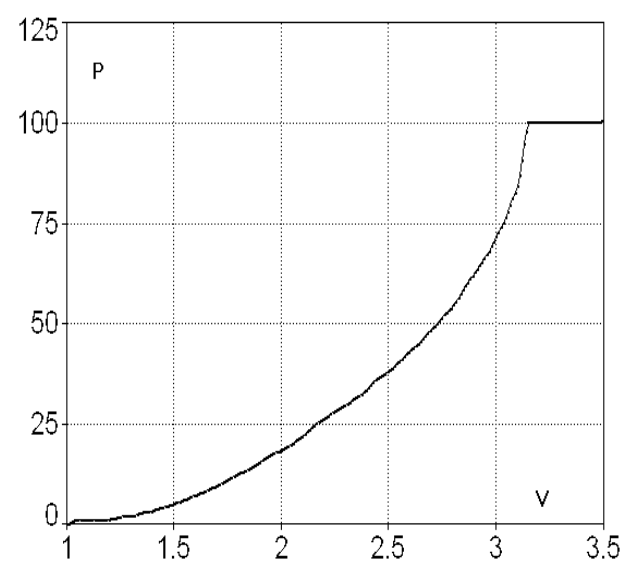

Figure 2. The number of particles (percentage $p$ ), passing two barriers in respect of particles velocity (autonomous equation)

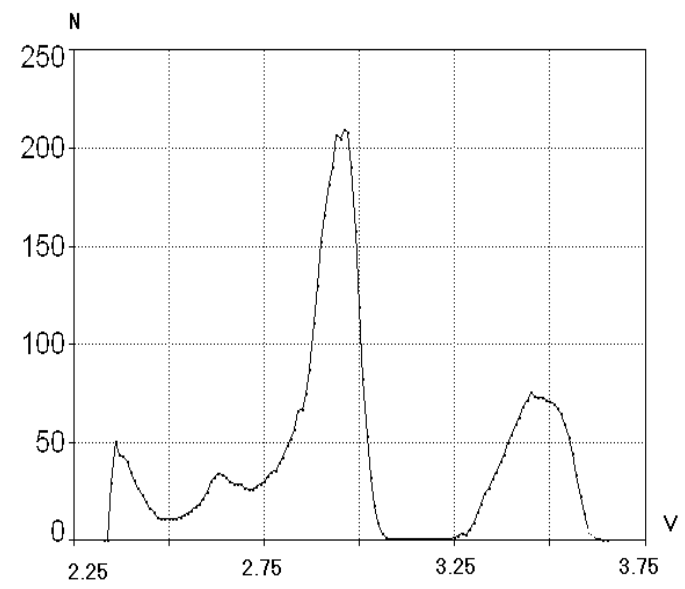

Figure 3. Distribution of particles in respect of velocity after passing two barriers (autonomous equation)

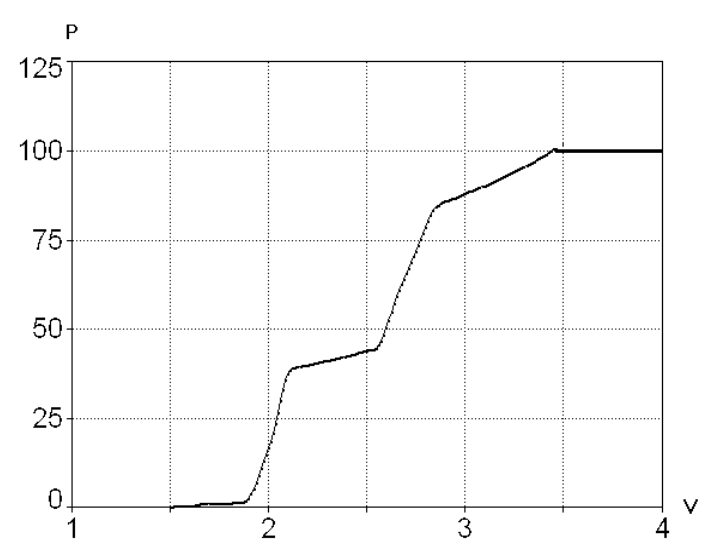

Figure 4. Probability of passing two barriers in respect of particles' velocity (non-autonomous equation) 


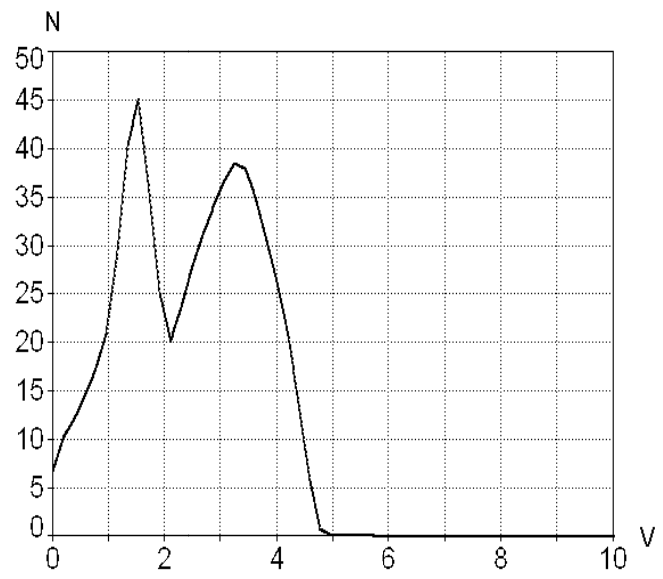

Figure 5. Distribution of particles in respect of velocities after passing two barriers non-autonomous equation

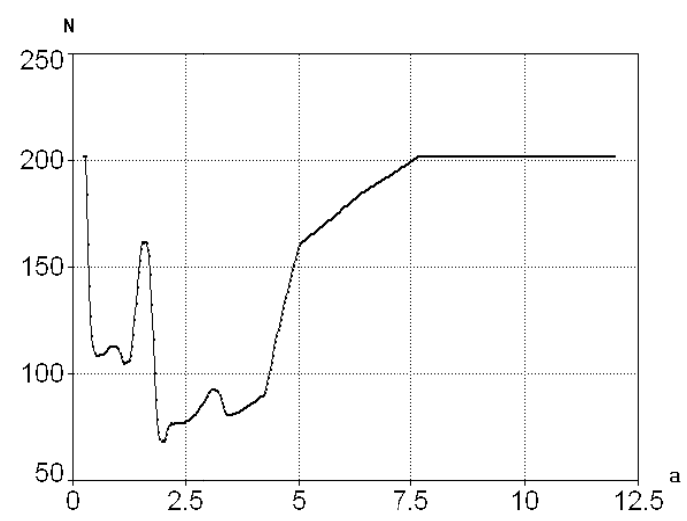

Figure 6. Number of particles, passing the barriers in respect of distance between barriers (non-autonomous equation)

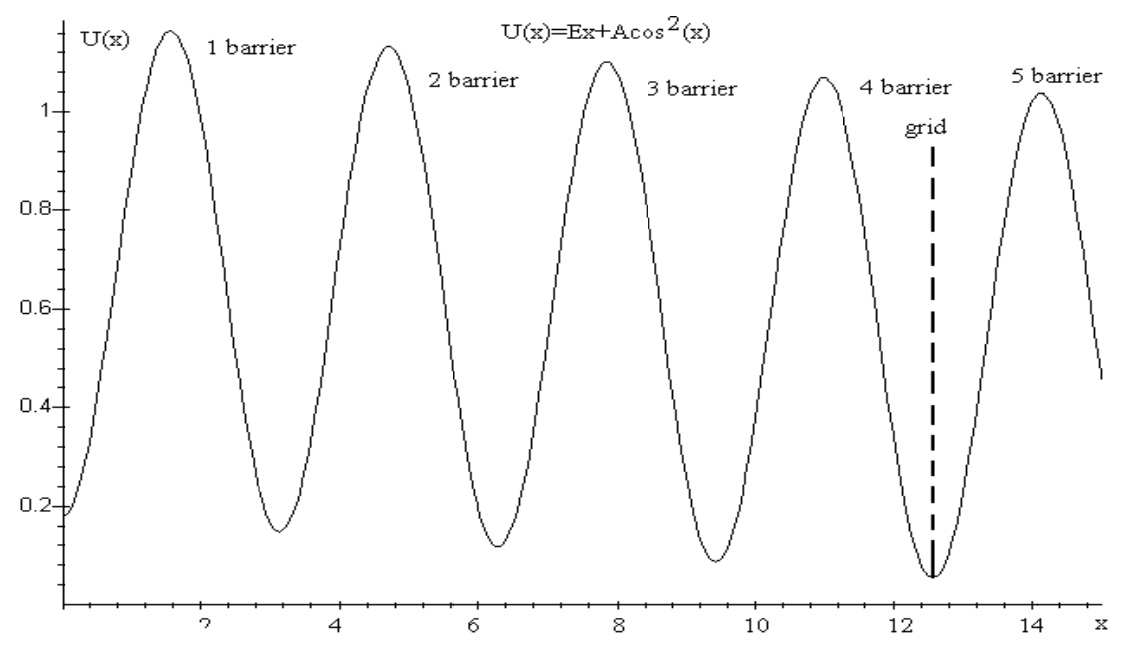

Figure 7. The Potential Barriers 


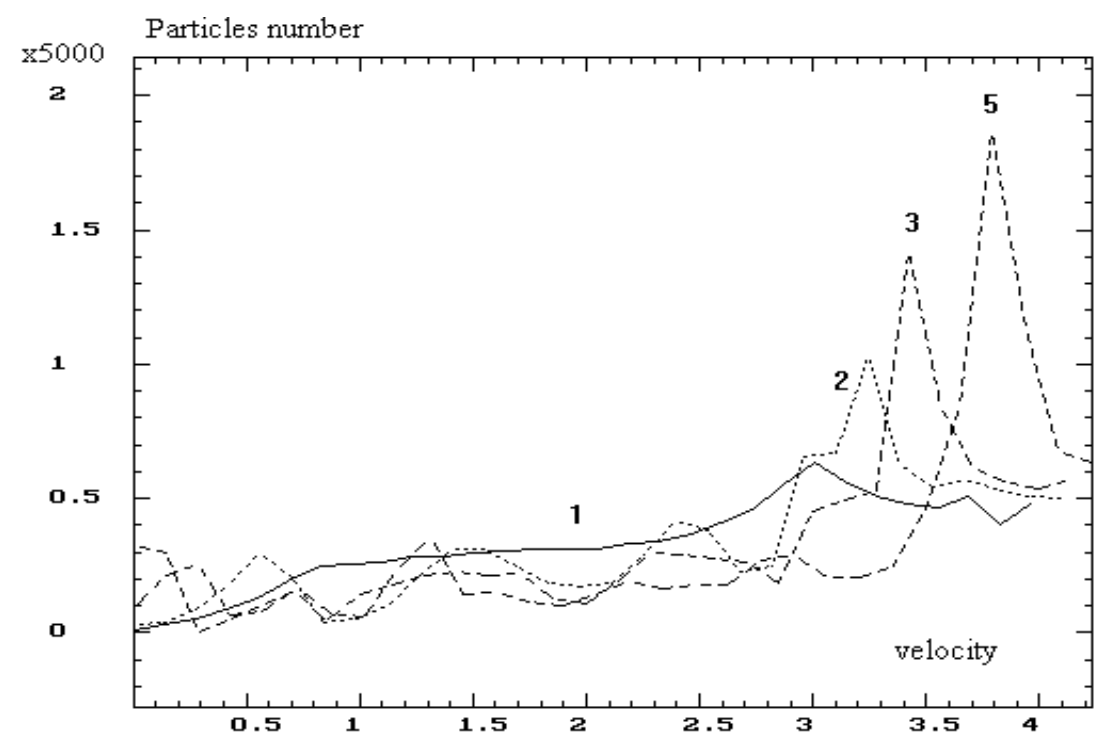

Figure 8 . Dependence of passed particles for different barriers number and velocity

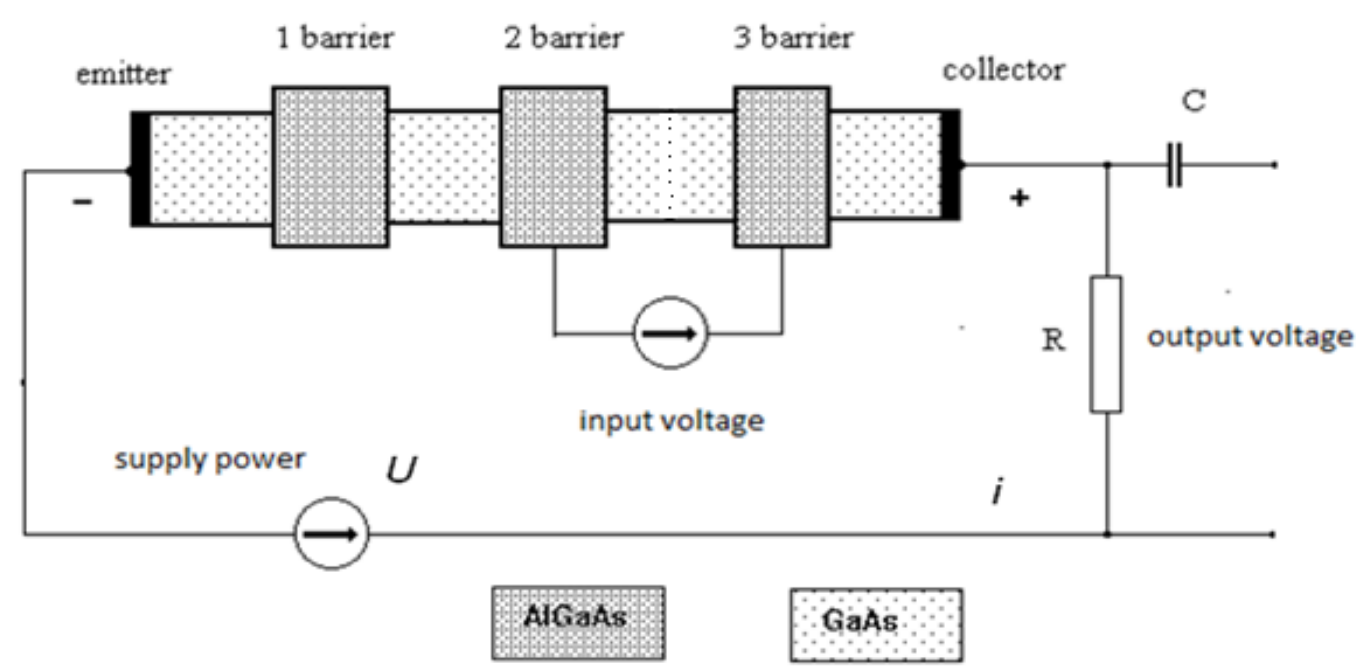

Figure 9.This is a way of possible realization of a new electronic device

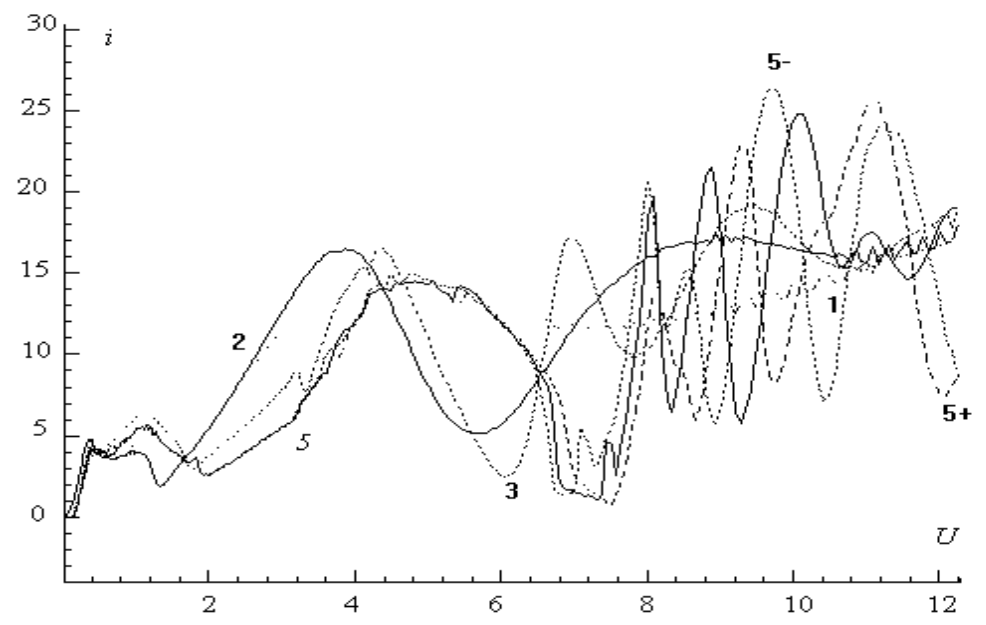

Figure 10. Voltage-current characteristic of a new device 